\title{
CT Image under Improved Fuzzy C-Means Clustering Algorithm for Evaluation of the Relationship between Cerebrospinal Fluid Change and Communicating Hydrocephalus after Decompressive Craniectomy in Patients with Traumatic Brain Injury
}

\author{
Yanmin Zhang $\mathbb{D}^{1},{ }^{1}$ Chunyue Zhang $\mathbb{D}^{2},{ }^{2}$ and Yan Wang $\mathbb{D}^{1}$ \\ ${ }^{1}$ Department of Neurosurgery, Hengshui People's Hospital, Hengshui 053000, Hebei, China \\ ${ }^{2}$ Department of Oncology, Hengshui People's Hospital, Hengshui 053000, Hebei, China \\ Correspondence should be addressed to Yanmin Zhang; 201309020550@stu.sdnu.edu.cn
}

Received 8 September 2021; Revised 10 January 2022; Accepted 12 January 2022; Published 9 February 2022

Academic Editor: M Pallikonda Rajasekaran

Copyright $\odot 2022$ Yanmin Zhang et al. This is an open access article distributed under the Creative Commons Attribution License, which permits unrestricted use, distribution, and reproduction in any medium, provided the original work is properly cited.

\begin{abstract}
This study was aimed to realize the automatic segmentation of communicating hydrocephalus lesions in brain CT after decompressive craniotomy (DC) in patients with traumatic brain injury (TBI) and discover correlation between cerebrospinal fluid changes and communicating hydrocephalus. Based on the traditional fuzzy C-means (FCM) algorithm, a new segmentation method filter-based FCM (FBFCM) algorithm was proposed. With 56 TBI patients as the research objects, the hydrocephalus lesions in CT images of patients after DC were segmented. The segmentation success rate (SSR), the segmentation coefficient $E_{p c}$, the segmentation entropy $E_{p e}$, and the number of iterations were indicators reflecting segmentation performance of FBFCM. The region of interest (ROI) on the segmented image was used to study the patient's cerebral aqueduct, foramen magnum, and C2 level of the cerebrospinal fluid velocity and flow, to analyze the characteristics of the occurrence of communicative hydrocephalus. It was indicated that average $E_{p c}$ of FBFCM algorithm was $0.9321 \pm 0.0144, E_{p e}$ was $0.1126 \pm 0.0081$, the average number of iterations was $14.42 \pm 3.79$, and the segmentation success rate was $96 \%$. Moreover, the above four indicators had statistically considerable differences compared with those of the FCM algorithm and the hard clustering algorithm $(\mathrm{HCM})(P<0.05)$. Analysis of the cerebrospinal fluid flow rate in patients with communicative hydrocephalus found that the cerebrospinal fluid flow rate at the midbrain aqueduct of the patient increased greatly. The net flow was $0.000 \pm 0.004 \mathrm{~mL} / \mathrm{s}$ in the aqueduct of the midbrain, $0.001 \pm 0.006 \mathrm{~mL} / \mathrm{s}$ in the foramen magnum, and $0.002 \pm 0.004 \mathrm{~mL} / \mathrm{s}$ in the $\mathrm{C} 2$ layer. In summary, the FBFCM algorithm is effective in the segmentation and processing of CT images, which can further improve the effect of this diagnosis. After examination, it is concluded that the cranial cerebrospinal fluid flow rate and flow of TBI patients are improved after DC treatment, so that the patient's condition can be effectively relieved. It has promotion value with clinical application.
\end{abstract}

\section{Introduction}

Traumatic brain injury (TBI) is caused by traffic accidents and various factors in everyday life $[1,2]$. After TBI, most patients chose conservative treatment [3-5], but when intracranial pressure (ICP) was consistently higher than $20-25 \mathrm{mmHg}$, decompressive craniotomy (DC) should be performed to remove intracranial hematoma and necrotic brain tissue to reduce intracranial pressure in patients with
CT showing large intracranial hematoma, midline displacement, and ring cistern closure [6,7]. Under normal circumstances, the secretion and absorption of cerebrospinal fluid maintain a dynamic balance, but when this balance is broken, such as increased intracranial pressure, excessive secretion of cerebrospinal fluid, or incomplete absorption of cerebrospinal fluid, there will be more and more accumulation of cerebrospinal fluid, resulting in dynamic expansion of the ventricular system and hydrocephalus formation. 
Posttraumatic hydrocephalus (PTH) is one of the most common complications after DC in TBI patients [8]. Clinical studies suggested that the pathogenesis of PTH is due to the oversecretion of cerebrospinal fluid after DC, which leads to abnormal changes in cerebrospinal fluid circulation dynamics and ultimately leads to the massive accumulation of cerebrospinal fluid in the ventricular or intracranial subarachnoid space [9]. At present, CT and MRI are mainly used as auxiliary examinations for hydrocephalus. MRI can clarify the diagnosis, classification, and cause of hydrocephalus, which can also make surgical plans and evaluate postoperative efficacy successively. The MRI manifestations of normal cranial hypertensive hydrocephalus are the narrowing of the convex and medial subarachnoid space of the brain, the enlargement of the ventricular system, and the widening of the lateral cleft cistern [10]. However, conventional MRI could not diagnose obstruction in water pipes, adhesion of basal cisterna, etc., which are just the main cause of communicating hydrocephalus [11]. CT mainly shows the enlargement of the lateral ventricle, the third ventricle, and the fourth ventricle, as well as the widening of the bilateral frontal angle with a size of more than $2 \mathrm{~cm}$. Temporal horn and aqueduct are seen, and the third ventricle is dilated, so CT is still the main method for the examination of communicating hydrocephalus [12]. However, there is noise and tissue overlap in the brain CT image, and the location of edema is uncertain. The similarity between the lesions and the white matter will make the diagnosis difficult for clinicians [13], thus affecting the clinical treatment of communicating hydrocephalus.

In recent years, computer-aided medical image analysis has obvious advantages in major breakthroughs in technology and improvement of medical service efficiency and capacity [14]. Segmentation means automatic or semiautomatic extraction of target regions from images for clinicopathological studies and disease diagnosis. However, as the current medical images output by complex imaging instruments are with massive miscellaneous medical information, the judgment accuracy of clinicians is affected in manual or semiautomatic methods, resulting in an increase in the rate of false detection [15]. Due to the above reasons, intelligent algorithm was employed to segment CT images, while fuzzy C-means (FCM) clustering algorithm was proved to have good effect in image segmentation after a large number of studies and analyses $[16,17]$. However, the consideration of this algorithm is not comprehensive enough, and the noise tolerance is poor, resulting in the reduction of the segmentation effect of the FCM algorithm [18]. Therefore, this study will improve and optimize the FCM algorithm and construct a lesion segmentation model based on the improved FCM algorithm, in order to explore the relationship between cerebrospinal fluid changes and communicating hydrocephalus after DC in TBI patients. It aimed to provide data reference for post-DC brain image recognition and lesion diagnosis in TBI.

\section{Methods}

2.1. Experimental Objects. A total of 56 patients with TBI treated in hospital from June 2018 to May 2019 were recruited. There were 38 males with an average age of
$45 \pm 10.12$ years and 18 females with an average age of $44.28 \pm 9.16$ years. Among the 56 patients, 29 were injured by car accident, 17 were injured by falling from height, and 10 were injured by hitting. All patients were admitted to hospital within $24 \mathrm{~h}$ after injury and received unilateral standard DC. All patients had signed the informed consent form, and this study had been approved by the ethics committee of the hospital.

2.2. Inclusion and Exclusion Criteria. Inclusion criteria were as follows: (i) patients aged between 18 and 65 years old, had a certain history of trauma, and had a certain organic TBI confirmed by CT and (ii) patient required surgical treatment with unilateral standard DC.

Exclusion criteria were as follows: (i) patient who had a history of TBI surgery, hydrocephalus, cerebrovascular disease, and so on; (ii) intracranial infection occurred after admission; and (iii) patient who had severe heart, brain, kidney, and lung diseases.

2.3. Surgical Methods. All preoperative preparations were completed after admission for all TBI patients, and contraindications for surgery were excluded. Conventional drug treatment was given before operation. According to the pathological characteristics of the patients, standard traumatic large bone flap surgery was performed. The patient was cut $1 \mathrm{~cm}$ in front of the tragus above the zygomatic arch, which was extended to the midline of the parietal bone from the upper and posterior direction of the auricle and then along the midline forward to the forehead under the hairline. Free bone flap or bone flap with temporal muscle was used. The range of bone flap was $10 \sim 12 \times 10 \sim 12 \mathrm{~cm}$. The patient's epidural hematoma, subdural hematoma, and intracerebral hematoma were removed. After the clearance, the temporalis fascia or artificial dura was removed for relaxation suture, and the scalp was sutured to complete the operation.

2.4. Implementation of CT Image Segmentation Method Based on FCM Algorithm. When the image is segmented, due to the differences of individual patients and the influence of imaging equipment, the obtained medical images usually have the characteristics of complexity and particularity. The difficulty of segmentation is increased when the image is segmented. FCM algorithm is the most classic fuzzy theory. According to ambiguity of the real data, FCM algorithm segments the image without any human intervention, which avoids threshold manually set in advance [19]. Fuzzy sets are different from classic sets that are expressed as follows.

$$
H_{A}(x)= \begin{cases}1, & x \in A \\ 0, & x \notin A\end{cases}
$$

In the above equation, $H_{A}(x)$ is the characteristic function of the set $A$. When $x$ is in it, the function is 1 , and it is 0 when $x \notin A$. The above equation shows that the element $x$ can only belong to a certain set, but the boundary of the set is fuzzy, so the set has the characteristic of "this and that." In general, a membership is established for each element and 
set. Each $x$ of the sets is mapped to $H_{A}(x)$ on interval $[0,1]$ by $H_{A}$. The larger $H_{A}(x)$, the greater the possibility of $x$ belonging to $A$. The smaller $H_{A}(x)$, the smaller the possibility of $x$ belonging to $A$. Classical set is a special kind of fuzzy set. When universe of discourse is a discrete finite set, there is the following equation.

$$
X=\left\{x_{1}, x_{2}, x_{3}, \cdots x_{n-1}, x_{n}\right\}
$$

The fuzzy set is as follows.

$$
A=\left\{H_{A}\left(x_{1}\right), H_{A}\left(x_{2}\right), \cdots H_{A}\left(x_{n}\right)\right\} .
$$

Each element $x$ of fuzzy set has a corresponding degree function $H_{A}\left(x_{i}\right)$. Zadeh's study is as follows.

$$
A=\left\{H_{A}(x) \mid x \in X\right\} .
$$

The pairwise notation is as follows.

$$
A=\left\{\left(H_{A}\left(x_{A}\right), x_{1}\right),\left(H_{A}\left(x_{A}\right), x_{2}\right), \cdots,\left(H_{A}\left(x_{A}\right), x_{n}\right)\right\} .
$$

The simple membership is as follows.

$$
A=\left\{H_{A}\left(x_{1}\right), H_{A}\left(x_{2}\right), \cdots, H_{A}\left(x_{n}\right)\right\} .
$$

The FCM algorithm uses iterative optimization of the objective function and sets a certain degree of each sample regarding membership function. The following matrix is the membership relationship between sample and category.

$$
U=\left[\begin{array}{cccc}
H_{11}, & H_{12}, & \cdots, & H_{1 z} \\
H_{21}, & H_{22}, & \cdots, & H_{2 z} \\
\cdots & & \\
H_{c 1}, & H_{c 2}, & \cdots, & H_{c z}
\end{array}\right] .
$$

In calculation, the constraints in (8) should be satisfied.

$$
\sum_{i=1}^{c} A_{i l}=1,1 \leq l \leq z .
$$

Objective function of FCM is as follows.

$$
\begin{gathered}
f_{\mathrm{FCM}}(U, S)=\sum_{l=1}^{c} \sum_{i=1}^{z}\left(H_{\mathrm{il}}^{t}\right) d_{\mathrm{il}}^{2}, \\
d_{i l}^{2}=\left\|x_{i}-h_{i}\right\|^{2},
\end{gathered}
$$

In equations (8) and (9), $t$ is the fuzzy factor, which sets degree of fuzziness of cluster's fuzzy set samples among different classes. $\left\|x_{i}-h_{l}\right\|$ is a paradigm between feature vector and cluster center, and $H_{i l}^{t}$ is membership of pixel $x i$ that belongs to the lth cluster center. $d_{i l}^{2}$ is Euclidean spatial distance between sample $x i$ and cluster center, which must meet the following constraint.

$$
\sum_{l=1}^{c} H_{\mathrm{il}}=1, H_{\mathrm{il}} \in(0,1), 1 \leq i \leq n, 1 \leq l \leq c .
$$

The multiplier method is utilized by FCM to update objective function iteratively, and membership matrix update is illustrated as follows.

$$
H_{\mathrm{il}}=\frac{1}{\sum_{j=1}^{c}\left(d_{\mathrm{il}} / d_{\mathrm{jl}}\right)^{2 / t-1}} .
$$

Update function for calculation of cluster centers is expressed as follows.

$$
h_{i}=\frac{\sum_{l=1}^{z}\left(H_{\mathrm{il}}\right)^{t} x_{l}}{\sum_{l=1}^{z}\left(H_{\mathrm{il}}\right)^{t}}, 1 \leq i \leq c .
$$

When FCM is employed to segment the CT image of the brain of a TBI patient, an original image is first input. After that, ambiguity $t$ and number $c$ of clusters are determined. Iteration number is set as $T$. Matrix $U$ is initialized under constraints. Number of iterations is set to $T=T+1$ after the initialization, and cluster centers and the membership degree matrix are updated iteratively. Two adjacent cluster centers are compared after the update. Then, the difference between them is calculated. The operation ends if difference is lower than convergence accuracy or number of iterations exceeds the set maximum iterations. Cluster center and membership matrix before the end are output. Finally, the following equation determines the attribution of the pixels on CT image based on principle of maximum membership.

$$
C_{j}=\arg _{i} \max \left(H_{\mathrm{ij}}\right) \forall i, \forall j .
$$

FCM has a poor tolerance for noise. Image will inevitably be interfered by some noise in the actual segmentation process, resulting in poor segmentation effect of the FCM algorithm. Figure 1 shows the anti-noise effect of FCM.

To denoise for FCM, a spatial filter was incorporated. CT images of the brain of TBI patients were filtered by Gaussian filter and median filter, and the filter-based FCM (FBFCM) was proposed. FBFCM's objective function is expressed as follows.

$$
\begin{aligned}
f_{\mathrm{FBFCM}}= & \sum_{i=1}^{c} \sum_{(x, y) \in I} H_{i}^{t}(x, y)\left\|I(x, y)-h_{i}\right\|^{2}+\alpha \sum_{i=1}^{c} \sum_{(x, y) \in I} H_{i}^{t}(x, y) \sum_{(x, y) \in N_{j}}\left\|G(x, y)-h_{i}\right\|^{2}, \\
& +\beta \sum_{i=1}^{c} \sum_{(x, y) \in I} H_{i}^{t}(x, y) \sum_{(x, y) \in N_{j}}\left\|M(x, y)-h_{i}\right\|^{2} .
\end{aligned}
$$



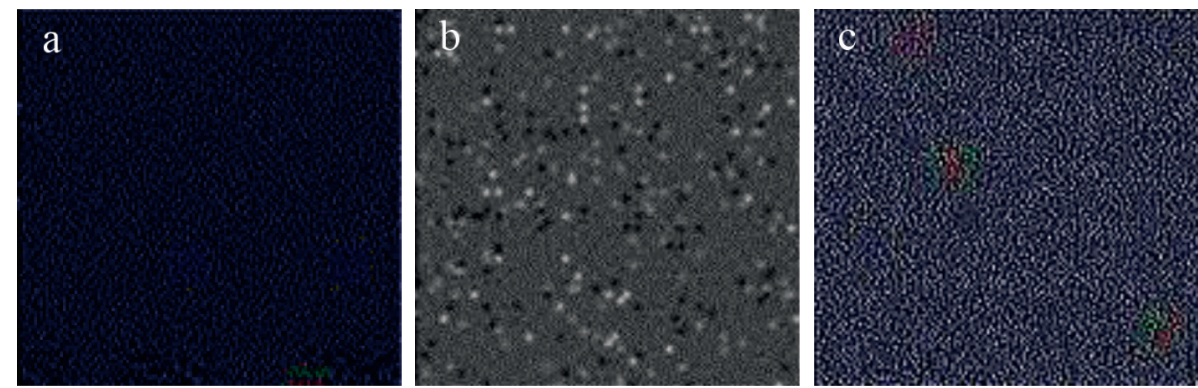

Figure 1: The clustering results of FCM. (a) The original image. (b) The image after addition of Gaussian noise. (c) The clustering result.

In equation (15), $c$ represent cluster centers, and $t$ is fuzzy weighting index of FBFCM. The larger $t$, the more obvious the smoothing effect. $I(x, y)$ is gray of pixel at point $(x, y)$ in original image. $N_{j}$ is neighborhood of current pixel, and $N_{R}$ is pixel neighborhood's size. $\alpha$ and $\beta$ are penalty factors, $h_{i}$ is cluster center set, $H_{i}^{t}(x, y)$ is set of fuzzy membership degrees of pixel $(x, y)$ to the $i$ th cluster center, and its function expression is as follows.

$$
\begin{aligned}
& \widehat{H}=\left\{h_{i}\right\},(i=1,2,3, \cdots, c), \\
& \widehat{H}=\left\{h_{i}\right\},(i=1,2,3, \cdots, c),
\end{aligned}
$$

The membership degree matrix and cluster center update are illustrated as follows.

$$
\begin{gathered}
h_{i}=\frac{\sum_{(x, y) \in I} H_{i}^{t}(x, y)[I(x, y)+\alpha G(x, y)+\beta M(x, y)]}{(1+\alpha+\beta) \sum_{(x, y)} H_{i}^{t}(x, y)}, \\
H_{i}(x, y)=\left(\sum_{l=1}^{c}\left(\frac{\left\|I(x, y)-h_{i}\right\|^{2}+\alpha\left\|G(x, y)-h_{i}\right\|^{2}+\beta\left\|M(x, y)-h_{i}\right\|^{2}}{\left\|I(x, y)-h_{l}\right\|^{2}+\alpha\left\|G(x, y)-h_{l}\right\|^{2}+\beta\left\|M(x, y)-h_{l}\right\|^{2}}\right)^{1 / m-1}\right)^{-1}, \\
i=1,2,3, \cdots, c .
\end{gathered}
$$

When the FBFCM algorithm is operated, the algorithm is initialized. Termination error threshold and the maximum number of iterations $T_{\max }$ are set. Cluster centers are extracted under iterative conditions after the CT image of the patient's brain was input.

The enhanced spatial filter is adopted to denoise the CT image of brain, and the filtered data $G(x, y)$ and $M(x, y)$ are recorded.

Cluster center and fuzzy membership matrix after denosing are updated by FBFCM. Two adjacent cluster centers are compared, and difference between the two is calculated. It is determined whether to end the operation regarding termination condition of FBFCM. After the algorithm runs, the obtained membership matrix receives threshold calculation, and it calculates the maximum of each row in matrix. Element of the maximum is set to 1 . Membership matrix is then obtained, and the brain CT image segmentation is completed.

2.5. Detection Indicators. To evaluate the effectiveness of the FBFCM algorithm for segmentation of CT images after DC, the segmentation success rate (SSR), segmentation coefficient $E_{p c}$, and segmentation entropy $E_{p e}$ were used for evaluation. The function expressions are as follows.

$$
\begin{aligned}
\operatorname{SSR} & =\frac{G_{\text {Correct }}}{N_{\text {Total }}} \times 100 \%, \\
E_{\mathrm{pc}} & =\frac{1}{N} \sum_{i=1}^{c} \sum_{(m, n) \in I} H_{i}^{t}(m, n), \\
E_{\mathrm{pe}} & =-\frac{1}{N} \sum_{i=1}^{c} \sum_{(m, n) \in I} H_{i}(m, n) \log H_{i}(m, n) .
\end{aligned}
$$

In equations (20)-(22), $G_{\text {Correct }}$ represents the number of images that are correctly segmented, and $N_{\text {Total }}$ is total number of images. In actual segmentation, the closer $E_{p c}$ is to 1 and the closer $E_{p e}$ is to 0 , the better the segmentation effect is. FCM algorithm and HCM algorithm were adopted for comparison. The pixel data in the ROI in each CT image of the patient were analyzed, and so was the flow of cerebrospinal fluid in the midbrain aqueduct and spinal canal.

2.6. Statistical Treatment. SPSS 22.0 was employed. Mean\pm standard deviation ( $\square x \pm s$ ) was the form of expression of measurement data. The $t$-test was adopted for data comparison between groups if data were normal distribution, while rank-sum test was used for data comparison if nonnormal distribution was not followed. If multiple sets of data 
were compared with normal distribution, analysis of variance was used. The difference was considerable if $P<0.05$.

\section{Results}

\subsection{Performance Analysis Based on Different Segmentation Algorithms}

3.1.1. Comparison of Segmentation Coefficient and Segmentation Entropy $E_{p e}$. In this study, the segmentation coefficient $E_{p c}$ and segmentation entropy $E_{p e}$ were calculated to objectively evaluate FBFCM. Manual segmentation results of clinical radiologists were used as the gold standard and were compared with others (Figure 2). The average $E_{p c}$ of the FCM algorithm was $0.8386 \pm 0.0651$, and $E_{p e}$ was $0.2255 \pm 0.0103$. The average $E_{p c}$ of the HCM algorithm was $0.8169 \pm 0.0778$, and $E_{p e}$ was $0.2831 \pm 0.0224$. The average $E_{p c}$ of FBFCM was $0.9321 \pm 0.0144$, and $E_{p e}$ was $0.1126 \pm 0.0081$. It was found that $E_{p c}$ and $E_{p e}$ of FBFCM were far superior to other algorithms $(P<0.05)$. By comparing the number of iterations of different algorithms, it was found that the average number of iterations of FBFCM was $14.42 \pm 3.79$, which was statistically different from the FCM algorithm and the HCM algorithm $(P<0.05)$.

\subsubsection{Comparison of Segmentation Time and Success Rate.} Different algorithms were used to segment the image, and the two parameters of segmentation time and segmentation success rate were recorded when the image was segmented. In Figure 3, the segmentation success rate of FBFCM was $96 \%$, and the average time was $15.11 \pm 3.29 \mathrm{~s}$, which was much lower than that of HCM algorithm. The FCM algorithm had a relatively shorter segmentation time but a lower segmentation success rate, and the HCM algorithm had the worst segmentation success rate and a longer time $(P<0.05)$. Therefore, FBFCM was more suitable for the segmentation of CT images of communicating hydrocephalus.

3.2. Image Filtering Processing. For the noise interference in the CT image of patients with TBI, the Gaussian filter and median filter in the FBFCM algorithm can filter the noise in the CT image, thereby suppressing the noise to the greatest extent. CT sequence images of one of the patients were selected to perform a filtering test, and the results are shown in Figure 4. The patient was a male with a severe open head injury due to a car accident. CT revealed bilateral temporal lobe epidural/subdural hematomas, multiple skull fractures, brain contusions, and subarachnoid hemorrhage. After admission, the preoperative examinations were performed, and so were craniotomy hematoma removal, craniectomy decompression, and skull defect repair in the emergency department under general anesthesia. The patient was cured after 40 days, and no obvious sequelae were found. Through filtering the CT images of the brain before and after the operation, it was found that the FBFCM algorithm can well retain important information such as the edge of the image while suppressing noise points.
3.3. FBFCM Algorithm Segmentation Results. Of the CT sequence images of communicating hydrocephalus in patients with TBI after DC, three randomly selected images were segmented using FBFCM algorithm to illustrate the accuracy of FBFCM algorithm segmentation. In Figure 5, the patient's CT showed that the ventricle was greatly enlarged. FBFCM algorithm was used to extract the patient's intracranial tissue, and then the intracranial tissue map was inverted to obtain the FBFCM cluster segmentation map. The results showed that when the gray level difference between the hydrocephalus area and the surrounding brain was small, FBFCM can still obtain good segmentation results.

3.4. CT Examination Results. CT examination was performed in 56 patients with TBI before decompression, and the pathological types of the patients were analyzed. The results are shown in Figure 6.

3.5. Correlation Analysis between Communicating Hydrocephalus and Changes in Cerebrospinal Fluid. A total of 7 cases of communicating hydrocephalus were diagnosed in 56 TBI patients after decompressive craniectomy. The patients' cerebral aqueduct, foramen magnum, and C2 level cerebrospinal fluid velocity and flow were calculated through the ROI in the image, and the results are shown in Figures 7 and 8 . The peak velocity and flow of cerebrospinal fluid at the level of cerebral aqueduct in patients with communicating hydrocephalus increased greatly, which was statistically different compared with normal patients $(P<0.05)$. The cerebrospinal fluid at the foramen magnum and the cerebrospinal fluid at the $\mathrm{C} 2$ level were smaller than those of normal patients, but there was no remarkable difference compared with normal patients $(P>0.05)$.

\section{Discussion}

Currently, it is believed that cerebrospinal fluid is mainly produced and secreted by choroid plexus tissues distributed on the ventricular wall [20-22]. Traffic hydrocephalus refers to the excessive accumulation of cerebrospinal fluid in the ventricular system or subarachnoid space [23]. In the treatment of TBI patients, tissue damage leads to increased cerebrospinal fluid secretion and increased intracranial pressure. Hydrocephalus is a dynamic process, and CT and MRI are often used for auxiliary examination. Segmentation means automatic or semiautomatic extraction of target regions from images for clinicopathological studies and disease diagnosis. However, as the current medical images output by complex imaging instruments are with massive miscellaneous medical information, the judgment accuracy of clinicians is affected in manual or semiautomatic methods, resulting in an increase in the rate of false detection. MRI is used to diagnose hydrocephalus, classify hydrocephalus, and identify the etiology, so as to formulate the surgical plan and evaluate the postoperative efficacy. However, there are noise and tissue overlap in brain CT images, the location of edema is uncertain, and the similarity 

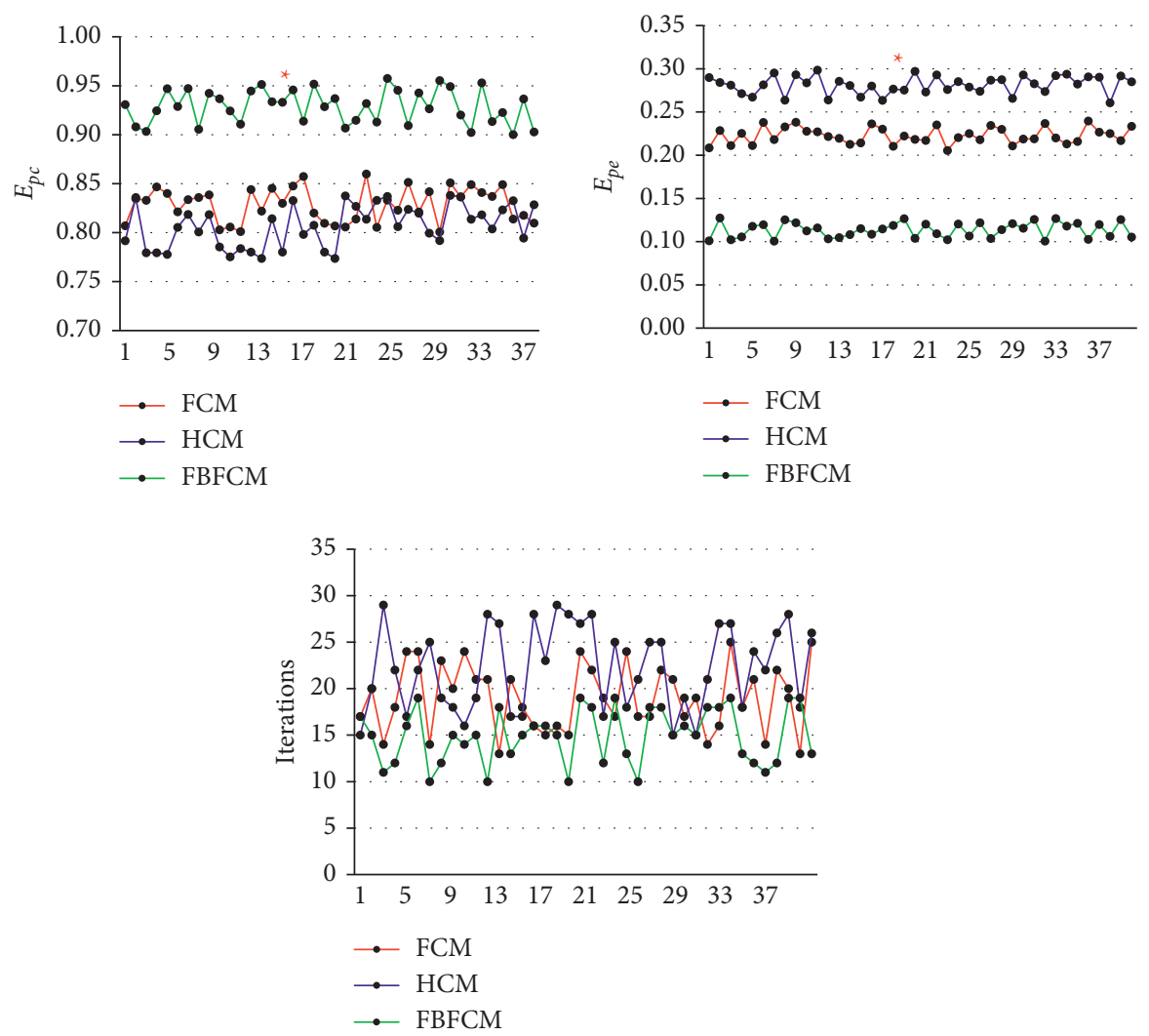

Figure 2: Objective analysis results of CT images based on different algorithms. “*” indicates that the difference had statistical significance $(P<0.05)$.
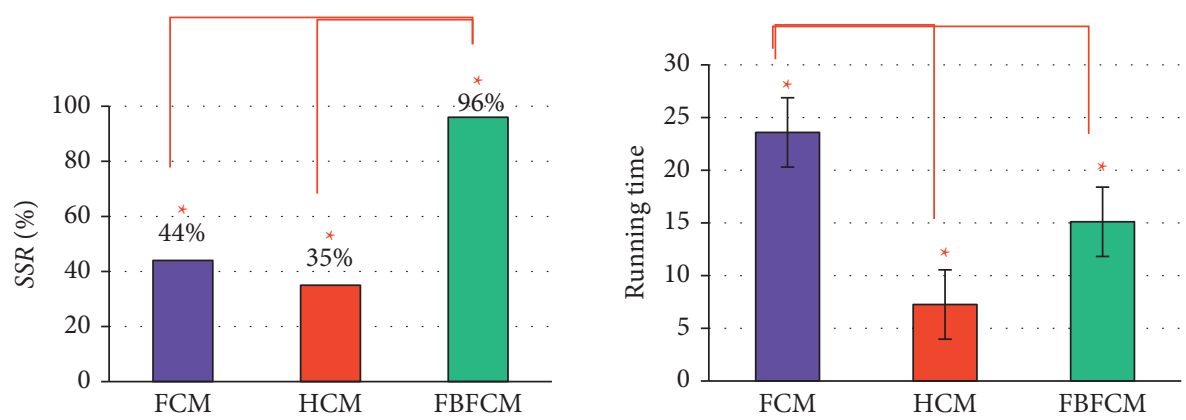

Figure 3: Comparison of lesion segmentation time and segmentation success rate of different algorithms. “ $*$ " indicates that the difference had statistical significance $(P<0.05)$.

between lesions and white matter brings difficulties to the diagnosis of clinicians. To solve this problem, FBFCM segmentation algorithm was used to optimize CT images in this study, and it was used for post-DC brain examination and diagnosis of TBI patients.

The results showed that $E_{p c}(0.9321 \pm 0.0144), E_{p e}$ $(0.1126 \pm 0.0081)$, and segmentation accuracy $(96 \%)$ of FBFCM algorithm were significantly better than those other algorithms. The average number of iterations of FBFCM $(14.42 \pm 3.79)$ was also lower than that of other algorithms $(P<0.05)$. The segmentation time of FCM $((7.26 \pm 2.78) \mathrm{s})$ was shorter than that of FBFCM $((15.11 \pm 3.29) \mathrm{s})$, but the segmentation accuracy was too low (35\%). In summary, the hydrocephalus region segmented by FBFCM algorithm was close to the real region, and the results were reproducible. In the case of a small amount of image data, the original image details were retained to the maximum extent. It was suitable for the segmentation of hydrocephalus lesions. The segmentation was completely automatic, and the segmentation results were accurate and reliable. It also indicated that Gaussian filter and median filter were effective in improving FCM algorithm. Tamal [24] proposed in their study that the segmentation algorithm using Gaussian filter can improve the reproducibility, robustness, and accuracy of segmentation. Pollnow et al. [25] pointed out in their study that Gaussian filter is a suitable tool for studying fluorescence optical data with low SNR. Pandey et al. [26] proved that median filter improved image quality. Wiharto and Suryani 


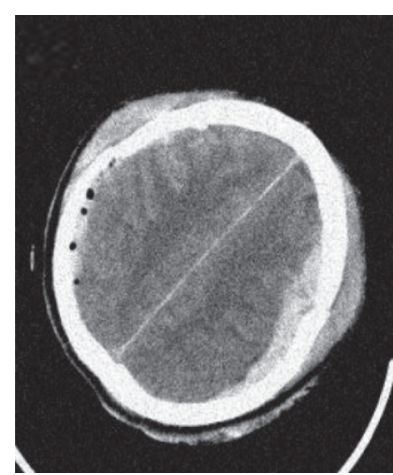

(a)

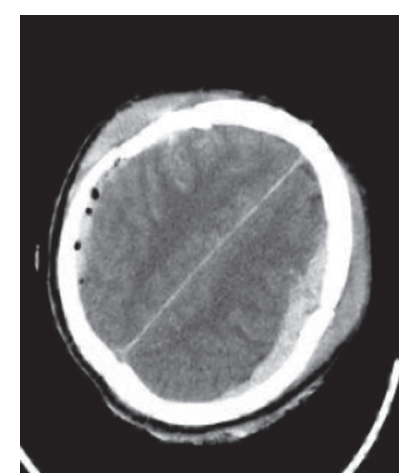

(b)

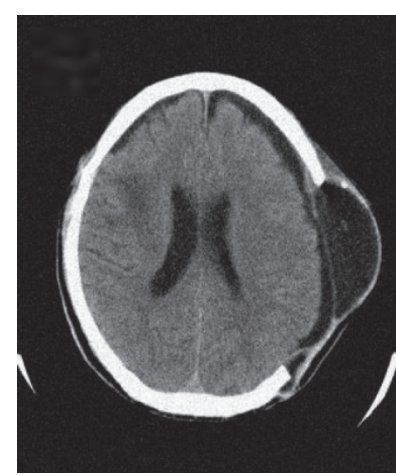

(c)

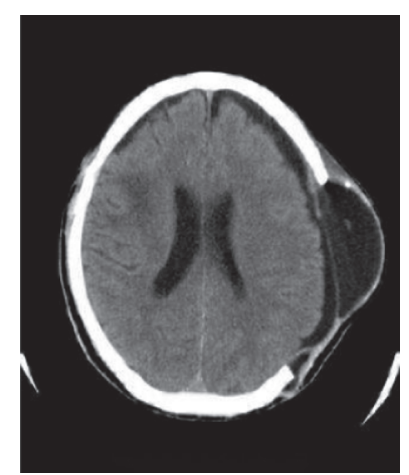

(d)

FIGURE 4: FBFCM algorithm filtering results. (a) The preoperative original image. (b) The preoperative filtered image. (c) The postoperative original image. (d) The postoperative filtered image.
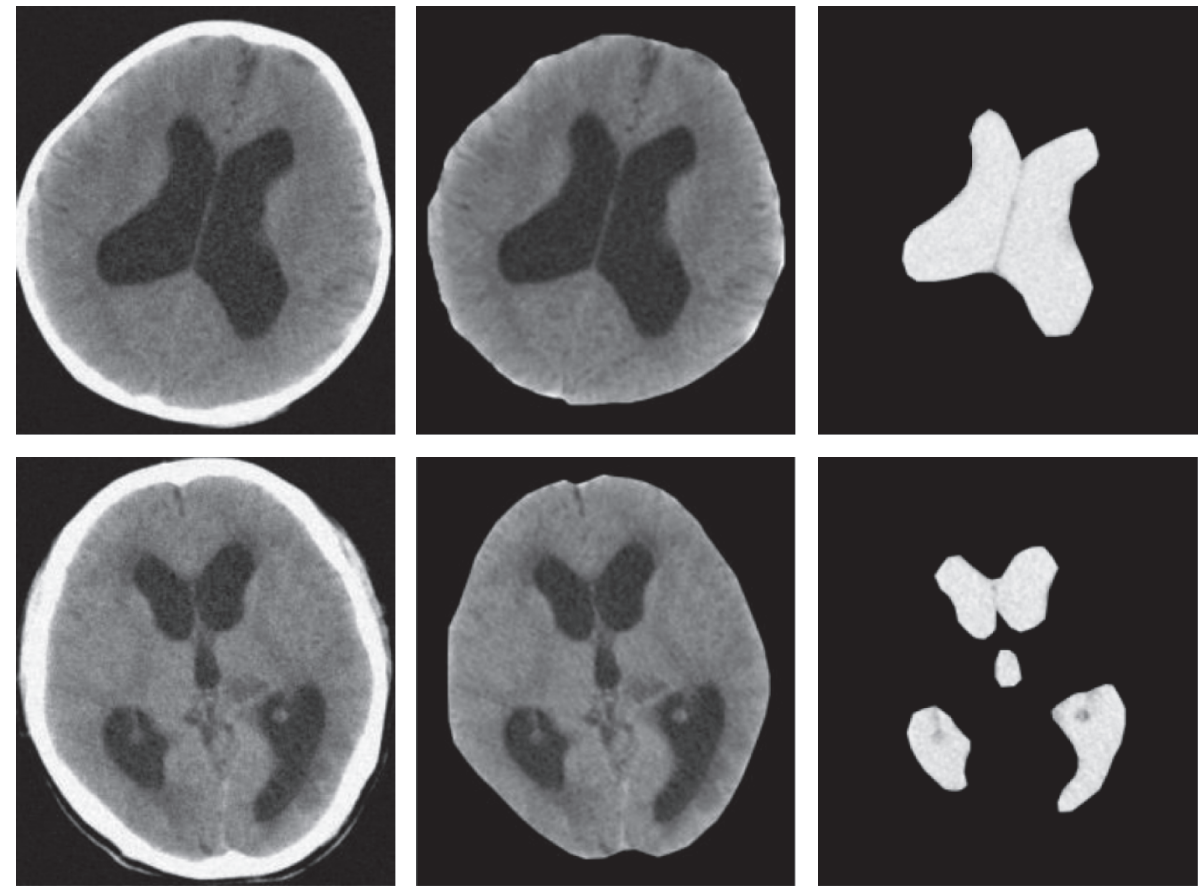

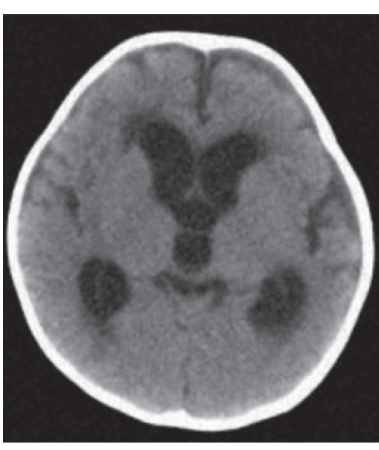

Original image

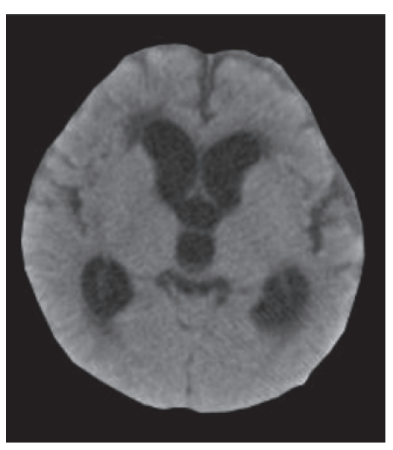

Brain tissue extract results

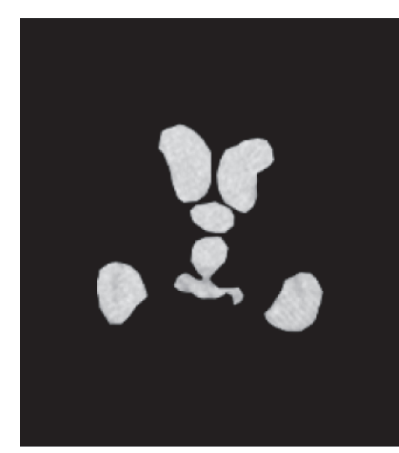

Segmentation results

Figure 5: Segmentation results of FBFCM algorithm. (a) Segmentation results. (b) Brain tissue extract results. (c) Original image.

[27] used median filter to optimize FCM in their study, and the results showed that the segmentation effect was significantly improved. The velocity and flow of cerebrospinal fluid in foramen magnum and C2 were lower than those in normal patients, but there was no statistical difference $(P>0.05)$. Those were similar to the research conclusions of 


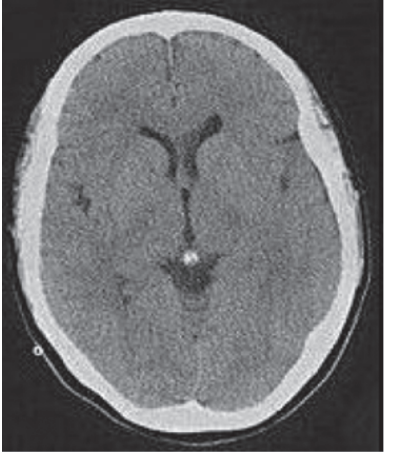

(a)

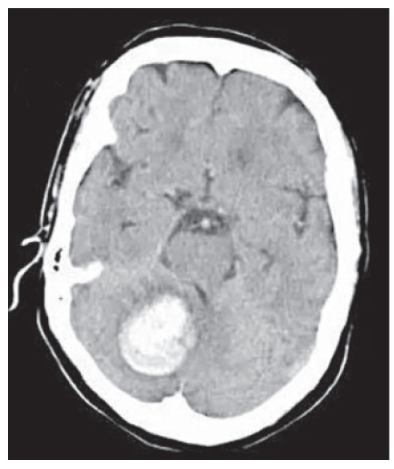

(d)

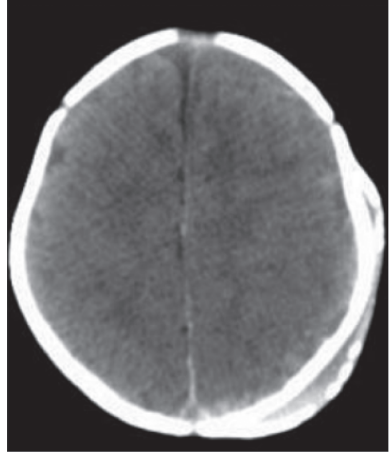

(b)

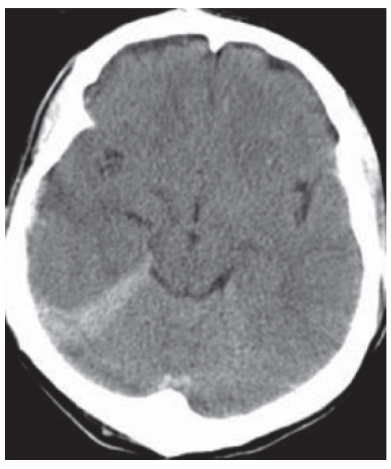

(e)

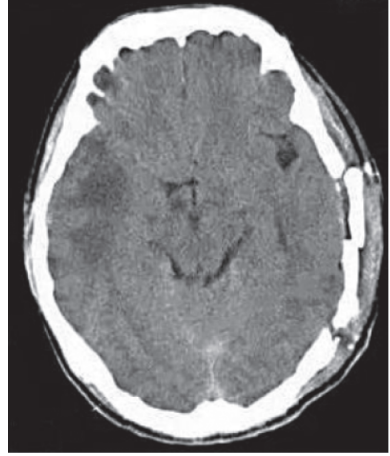

(c)

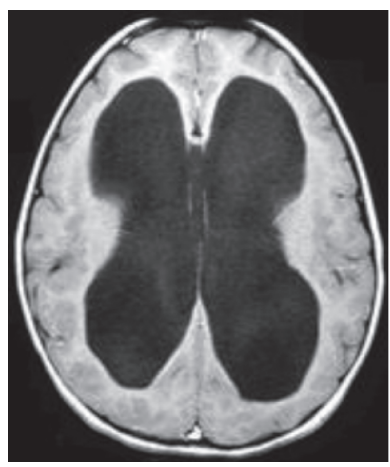

(f)

FiguRE 6: Examples of CT images of some patients' brains. (a) Normal brain. (b) Scalp injury. (c) Skull fracture. (d) Subdural hematoma. (e) Subdural hematoma. (f) Hydrocephalus.
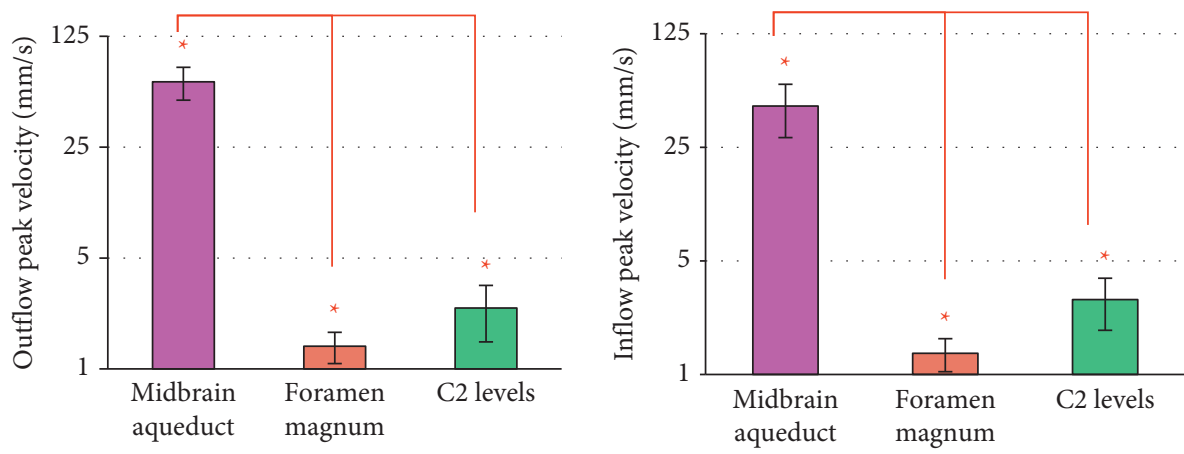

Figure 7: Flow velocity of cerebrospinal fluid at the midbrain aqueduct, foramen magnum, and C2 in communicating hydrocephalus. “*” indicates that the difference had statistical significance $(P<0.05)$.
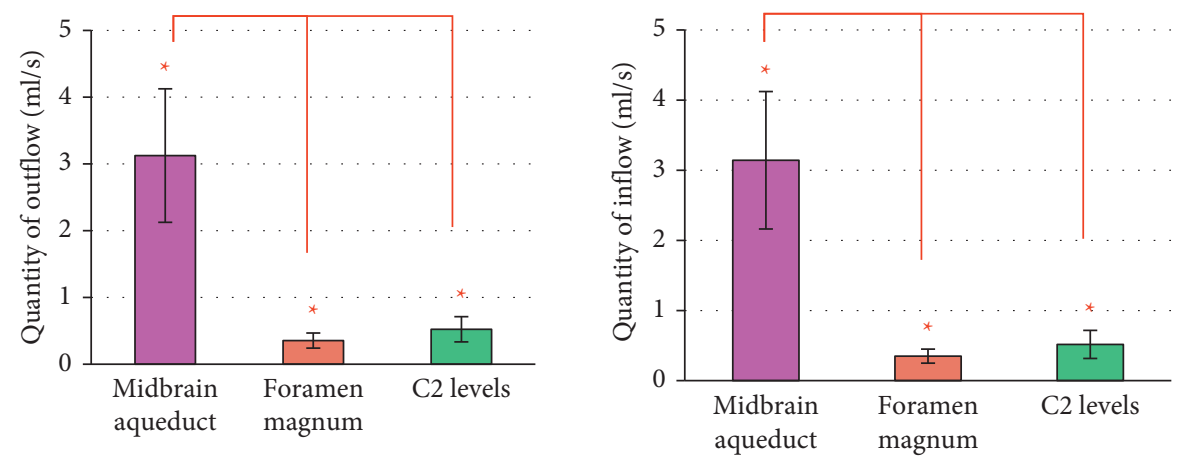

FIgURE 8: Comparison of the flow of cerebrospinal fluid at the midbrain aqueduct, foramen magnum, and C2 in communicating hydrocephalus. “*” indicates that the difference had statistical significance $(P<0.05)$. 
Lalou et al. [28]. It may be because the subarachnoid space compliance of patients with communicating hydrocephalus was reduced, resulting in weakened arterial pulsation and increased intracranial pulse pressure, thus expanding the ventricular system. The simultaneous reduction of arterial pulsation led to a decrease in cerebrospinal fluid volume, a decrease in the pressure on the cortical vein into the dural sinus, and a decrease in arterial expansion causing decrease in cerebral blood flow.

\section{Conclusion}

In this study, CT images based on improved FCM segmentation algorithm were used for post-DC brain examination and diagnosis of TBI patients, and its application value was evaluated. Then, the correlation between postoperative cerebrospinal fluid changes and traffic-induced hydrocephalus was analyzed and studied. The results showed that FBFCM has a significant application effect in the segmentation processing of $\mathrm{CT}$ images, which can further improve the effect of the diagnosis. After examination, it is concluded that the cranial cerebrospinal fluid flow rate and flow of TBI patients are improved after DC treatment, so that the patient's condition can be effectively relieved. It has promotion value with clinical application. However, this study still has some limitations, which are reflected in the relatively low incidence of cases in a single medical institution. In addition, due to the limitations of various inclusion conditions, the overall sample size is relatively small, and the results of statistical analysis from different perspectives may have large errors, which will be addressed in the future work.

\section{Data Availability}

The data used to support the findings of this study are available from the corresponding author upon request.

\section{Conflicts of Interest}

The authors declare that they have no conflicts of interest.

\section{References}

[1] B. Dang, W. Chen, W. He, and G. Chen, "Rehabilitation treatment and progress of traumatic brain injury dysfunction," Neural Plasticity, vol. 2017, Article ID 1582182, 6 pages, 2017.

[2] K. K. Wang, Z. Yang, T. Zhu et al., "An update on diagnostic and prognostic biomarkers for traumatic brain injury," Expert Review of Molecular Diagnostics, vol. 18, no. 2, pp. 165-180, 2018.

[3] M. Galgano, G. Toshkezi, X. Qiu et al., "Traumatic brain injury," Cell Transplantation, vol. 26, no. 7, pp. 1118-1130, 2017.

[4] R. M. Jha, P. M. Kochanek, and J. M. Simard, "Pathophysiology and treatment of cerebral edema in traumatic brain injury," Neuropharmacology, vol. 145, no. Pt B, pp. 230-246, 2019.

[5] M. L. Pearn, I. R. Niesman, J. Egawa et al., "Pathophysiology associated with traumatic brain injury: current treatments and potential novel therapeutics," Cellular and Molecular Neurobiology, vol. 37, no. 4, pp. 571-585, 2017.

[6] E. J. Barthélemy, M. Melis, E. Gordon, J. S. Ullman, and I. M. Germano, "Decompressive craniectomy for severe traumatic brain injury: a systematic review," World Neurosurgery, vol. 88, pp. 411-420, 2016.

[7] J. Sahuquillo and J. A. Dennis, "Decompressive craniectomy for the treatment of high intracranial pressure in closed traumatic brain injury," Cochrane Database of Systematic Reviews, vol. 12, no. 12, 2019.

[8] R. Fattahian, S. Bagheri, and M. Sadeghi, "Development of posttraumatic hydrocephalus requiring ventriculoperitoneal shunt after decompressive craniectomy for traumatic brain injury: a systematic review and meta-analysis of retrospective studies," Medical Archives, vol. 72, no. 3, pp. 214-219, 2018.

[9] G. Di, Q. Hu, D. Liu, X. Jiang, J. Chen, and H. Liu, "Risk factors predicting posttraumatic hydrocephalus after decompressive craniectomy in traumatic brain injury," World Neurosurgery, vol. 116, pp. e406-e413, 2018.

[10] R. Wu, Y. Ye, T. Ma, G. Jia, and H. Qin, "Management of subdural effusion and hydrocephalus following decompressive craniectomy for posttraumatic cerebral infarction in a patient with traumatic brain injury: a case report," $B M C$ Surgery, vol. 19, no. 1, p. 26, 2019.

[11] S. Langner, S. Fleck, J. Baldauf et al., "Of hydrocephalus in adults,” Röfo, vol. 189, no. 8, pp. 728-739, 2017.

[12] N. J. Modi, M. Agrawal, and V. D. Sinha, "Post-traumatic subarachnoid hemorrhage," A review. Neurol India, vol. 64, no. S8-S13, 2016.

[13] L. Walker, A. Gholamrezanezhad, D. Bucklan, P. F. Faulhaber, and J. K. O'Donnell, “SPECT/CT detection of a communicating arachnoid cyst in a patient with normal pressure hydrocephalus," Clinical Nuclear Medicine, vol. 42, no. 7, pp. 555-557, 2017.

[14] S. Guo, R. Chen, H. Li, T. Zhang, and Y. Liu, "Identify severity bug report with distribution imbalance by CR-SMOTE and ELM," International Journal of Software Engineering and Knowledge Engineering, vol. 29, no. 2, pp. 139-175, 2019.

[15] H. W. Goo, "Hydrocephalus: ventricular volume quantification using three-dimensional brain CT data and semiautomatic three-dimensional threshold-based segmentation approach," Korean Journal of Radiology, vol. 22, no. 3, pp. 435-441, 2021.

[16] H. Hanzouli-Ben Salah, J. Lapuyade-Lahorgue, J. Bert et al., "A framework based on hidden Markov trees for multimodal PET/CT image co-segmentation," Medical Physics, vol. 44, no. 11 , pp. 5835-5848, 2017.

[17] W. Wu, S. Wu, Z. Zhou, R. Zhang, and Y. Zhang, "3D liver tumor segmentation in CT images using improved fuzzy C-means and graph cuts," BioMed Research International, vol. 2017, Article ID 5207685, 11 pages, 2017.

[18] J. Zhang and Z. Ma, "Hybrid fuzzy clustering method based on FCM and enhanced logarithmical PSO (ELPSO)," Computational Intelligence and Neuroscience, vol. 2020, Article ID 1386839, 12 pages, 2020.

[19] S.-J. Peng, C.-C. Lee, H.-M. Wu et al., "Fully automated tissue segmentation of the prescription isodose region delineated through the Gamma knife plan for cerebral arteriovenous malformation (AVM) using fuzzy C-means (FCM) clustering," NeuroImage: Clinica, vol. 21, Article ID 101608, 2019.

[20] A. K. Filis, K. Aghayev, and F. D. Vrionis, "Cerebrospinal fluid and hydrocephalus: physiology, diagnosis, and treatment," Cancer Control, vol. 24, no. 1, pp. 6-8, 2017. 
[21] J. K. Karimy, J. Zhang, D. B. Kurland et al., "Inflammationdependent cerebrospinal fluid hypersecretion by the choroid plexus epithelium in posthemorrhagic hydrocephalus," $\mathrm{Na}$ ture Medicine, vol. 23, no. 8, pp. 997-1003, 2017.

[22] M. P. Lun, E. S. Monuki, and M. K. Lehtinen, "Development and functions of the choroid plexus-cerebrospinal fluid system," Nature Reviews Neuroscience, vol. 16, no. 8, pp. 445-457, 2015.

[23] A. Agarwal, G. Bathla, and S. Kanekar, "Imaging of communicating hydrocephalus," Seminars in Ultrasound, CT and MRI, vol. 37, no. 2, pp. 100-108, 2016.

[24] M. Tamal, "A phantom study to assess the reproducibility, robustness and accuracy of PET image segmentation methods against statistical fluctuations," PLoS One, vol. 14, no. 7, 2019.

[25] S. Pollnow, N. Pilia, G. Schwaderlapp, A. Loewe, O. Dössel, and G. Lenis, "An adaptive spatio-temporal Gaussian filter for processing cardiac optical mapping data," Computers in Biology and Medicine, vol. 102, pp. 267-277, 2018.

[26] A. K. Pandey, A. Sharma, P. D. Sharma et al., "Denoising of iodine-131 images using a median filter," Nuclear Medicine Communications, vol. 40, no. 4, pp. 308-316, 2019.

[27] W. Wiharto and E. Suryani, "The comparison of clustering algorithms K-means and fuzzy C-means for segmentation retinal blood vessels," Acta Informatica Medica, vol. 28, no. 1, pp. $42-47,2020$.

[28] A. D. Lalou, V. Levrini, M. Czosnyka et al., "Cerebrospinal fluid dynamics in non-acute post-traumatic ventriculomegaly," Fluids and Barriers of the CNS, vol. 17, no. 1, p. 24,2020 . 